\title{
Study of Tip-Induced Ti-Film Oxidation in Atomic Force Microscopy Contact and Non-Contact Mode
}

\author{
J. S̆oltÝs*, V. Cambel and J. Fedor \\ Institute of Electrical Engineering, Slovak Academy of Sciences \\ Dúbravská cesta 9, 84104 Bratislava, Slovakia
}

\begin{abstract}
Local anodic oxidation of metals using scanning probe techniques is mostly used for fabrication of isolated gates. The high-resistance oxide created in such a manner divides a thin metallic film into isolated regions. The tip-induced metallic oxide has not so far been used in nanolithography processes as a masking material. The aim of this contribution is to study the technological potential of a $\mathrm{TiO}_{x}$ mask prepared by the local anodic oxidation of a Ti film. Such a mask can be used to complete a nanotechnology process using atomic force microscopy, which can be easily combined with standard optical lithography techniques. We have found that the insulating properties of the oxides prepared in contact and non-contact modes differ strongly - they represent an energy barrier of $200 \mathrm{meV}$ and $400 \mathrm{meV}$, respectively.
\end{abstract}

PACS numbers: 07.79.Lh, 81.16.Nd

\section{Introduction}

The local anodic oxidation (LAO) technique using the atomic force microscope (AFM) is an effective tool for the fabrication of structures and devices on the nanometer scale [1]. The potential of the technique for fabrication of nanostructures has been recognized several years ago and has been realized in variety of approaches. Great advantage of the method is its universality - the technique can be applied directly to metals ( $\mathrm{Al}, \mathrm{Ti})$ as well as to semiconductors ( $\mathrm{GaAs}, \mathrm{Si}$ ). It can be combined with traditional resist exposition, with direct resist exposition by AFM-tip voltage, or with resist scratching of thin resist layers. All methods mentioned above are used for fabrication of quantum point contacts (QPC) [2] tuned by in-plane as well as by top gate biasing, for quantum wires realized by di-

*corresponding author; e-mail: eleksoto@savba.sk 
rect semiconductor oxidation, and for room-temperature single electron transistors (SETs) [3] with pronounced Coulomb oscillations.

LAO applied to semiconductors (GaAs/AlGaAs) is used to lower the carrier concentration in heterostructures below the oxidized region, so by writing two parallel lines a quantum wire can be easily defined [1]. Moreover, oxidized semiconductor can serve as an etching mask for further processing. The LAO of metals is mostly used for the fabrication of isolated gates, utilized, for example, in SETs. The technique is used to oxidize $\approx 5-10 \mathrm{~nm}$ thick metal films along the lines written by the tip. The high-resistance oxide created divides the metallic film into isolated regions that represent independent gates. But the tip-induced metallic oxide has not, so far, been used in nanolithography processes as a masking material.

The aim of this contribution is to study the technological potential of a $\mathrm{TiO}_{x}$ mask prepared by the LAO of a Ti film. Such a mask could be used to complete the nanotechnology process using AFM, which can be easily combined with standard optical lithography techniques. For this purpose we have studied the application possibilities of tip-induced local anodic oxidation of Ti films using AFM in contact and non-contact mode, under constant voltage applied to the tip. We explore also insulating properties of $\mathrm{TiO}_{x}$ prepared in both modes in planar metal-insulator-metal (MIM) diode configuration.

\section{TiO $_{x}$ mask preparation using LAO}

LAO of thin Ti films is an analogue of electrochemical anodic oxidation [4]. Lithography mode, humidity, tip speed, and applied voltage are the basic conditions that affect the structure of the Ti oxide formed. Usually, a positive potential is applied to the sample and a negative one to the tip.

We have tested the $\mathrm{TiO}_{x}$ etching mask prepared by LAO on GaAs substrate for future applications in GaAs nanotechnology. The technology can be divided into several steps. Figure 1 depicts the most important of them. During the step (a) a clean layer of $\mathrm{Ti}$ of the thickness $10 \mathrm{~nm}$ was evaporated onto (100)-oriented semi-insulating GaAs substrates in an ultra-high-vacuum evapora-

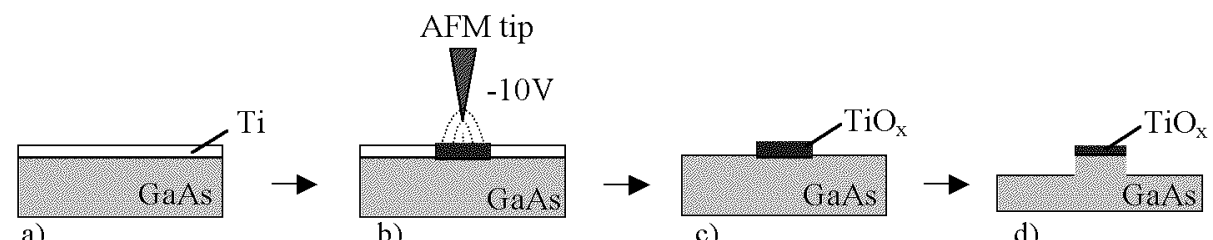

b)

c)

d)

Fig. 1. Schematic nanotechnology utilizing $\mathrm{TiO}_{x}$ as a mask prepared by LAO: (a) evaporation of Ti film ( $\approx 10 \mathrm{~nm}$ thickness), (b) local anodic oxidation of Ti using AFM either in the contact or in the non-contact mode, (c) etching off Ti layer in HF, (d) etching off GaAs using $\mathrm{TiO}_{x}$ as a mask. 
tion system. Then a periodic structure was defined by optical lithography in Ti using $\mathrm{HF}-\mathrm{H}_{2} \mathrm{O}$ solution. Contacts were prepared by standard contacting using $\mathrm{Au}$ wires directly onto the Ti layer. In step (b) LAO was applied to the structure (AFM Topometrix Explorer used) under the following conditions: applied voltage - $8 \mathrm{~V}$ in the contact mode or $13 \mathrm{~V}$ in the non-contact mode, humidity $40 \%$, tip speed $0.05 \mu \mathrm{m} / \mathrm{s}$. The lines were overwritten 10 times to ensure oxidation up to the GaAs substrate. In step (c) the unoxidized parts of the Ti layer were removed in $\mathrm{HF}-\mathrm{H}_{2} \mathrm{O}$ solution. Finally, $100 \mathrm{~nm}$ thick GaAs layer was etched off in $\mathrm{H}_{3} \mathrm{PO}_{4}-\mathrm{H}_{2} \mathrm{O}_{2}-\mathrm{H}_{2} \mathrm{O}$ solution [5].

It is was found that the resistance of $\mathrm{TiO}_{x}$ against etchants used depended strongly on the oxidation mode, most probably on the oxygen content in the titanium oxide formed. $\mathrm{TiO}_{x}$ suitable for masks in GaAs nanotechnology can be prepared in the contact mode only, which is documented by the following figures.

Figures 2 and 3 show typical AFM scans of $\mathrm{TiO}_{x}-$ GaAs structures prepared using LAO in the contact and in the non-contact modes, respectively. In Fig. 2a

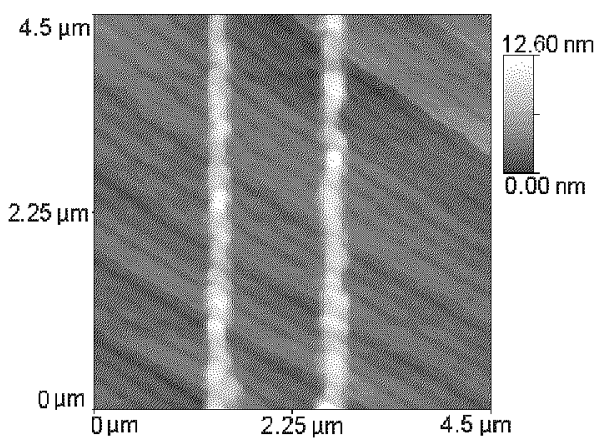

a)

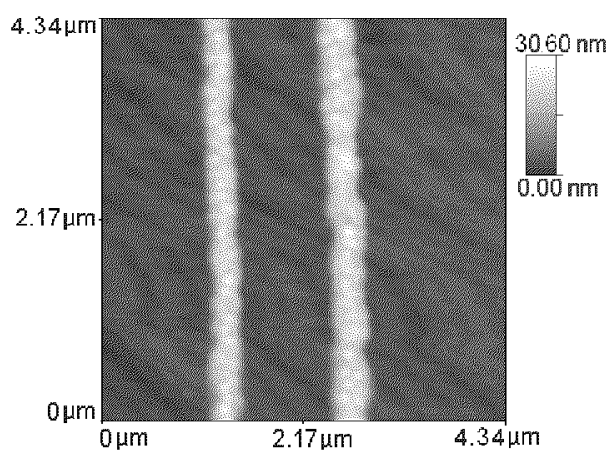

b)

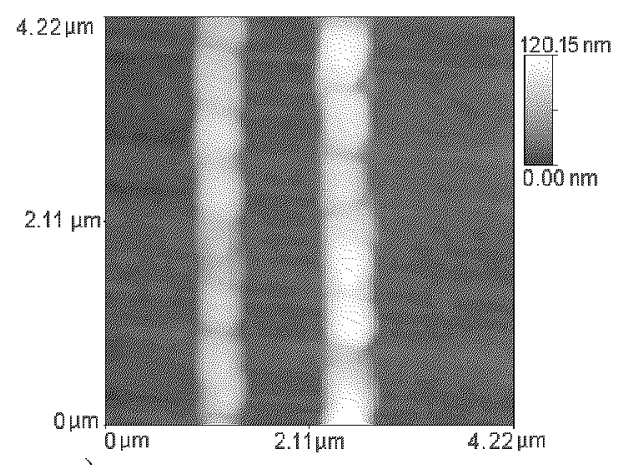

c)

Fig. 2. $\mathrm{TiO}_{x}$ lines written in the contact mode after: (a) local anodic oxidation of the lines, (b) removal of the Ti film in $\mathrm{HF}-\mathrm{H}_{2} \mathrm{O}$ solution, (c) $\approx 100 \mathrm{~nm}$ etch off of GaAs in $\mathrm{H}_{3} \mathrm{PO}_{4}-\mathrm{H}_{2} \mathrm{O}_{2}-\mathrm{H}_{2} \mathrm{O}$ solution. 


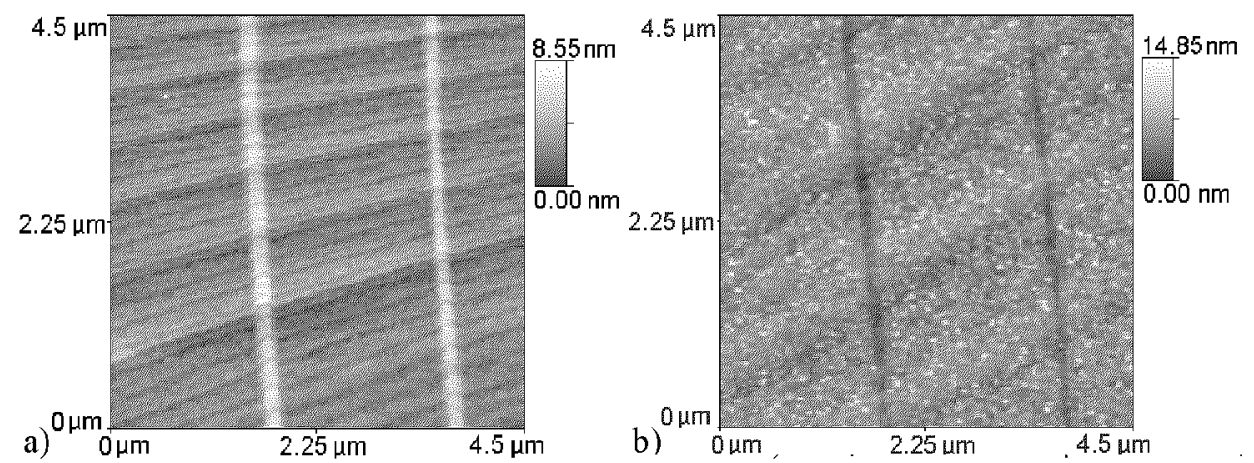

Fig. 3. $\mathrm{TiO}_{x}$ lines written in the non-contact mode after: (a) local anodic oxidation of the lines, (b) removal of the Ti film in $\mathrm{HF}-\mathrm{H}_{2} \mathrm{O}$ solution. Oxide lines have completely disappeared as well as the Ti film.

the AFM scan of the Ti surface directly after LAO is shown. Figure $2 \mathrm{~b}$ shows the scan after removal of the unoxidized Ti film in $\mathrm{HF}-\mathrm{H}_{2} \mathrm{O}$ solution, whereas Fig. 2c shows the scan after $100 \mathrm{~nm}$ deep etching of GaAs in $\mathrm{H}_{3} \mathrm{PO}_{4}-\mathrm{H}_{2} \mathrm{O}_{2}-\mathrm{H}_{2} \mathrm{O}$ solution. Here $\mathrm{TiO}_{x}$ lines remain resistant against the solution applied. In contrast, the oxide lines written in the non-contact mode (Fig. 3) were etched off during removal of $\mathrm{Ti}$ in $\mathrm{HF}-\mathrm{H}_{2} \mathrm{O}$ solution, although the remaining etching conditions remained the same. Several other etchants have been tested with the same result - oxide lines written in the non-contact mode were removed with any solution used to remove Ti.

\section{Electrical characterization of the $\mathrm{TiO}_{x}$ barrier}

For a better understanding of the experimental results presented above, we have prepared a special structure to test the insulating properties of $\mathrm{TiO}_{x}$ formed by the AFM tip in the contact and in the non-contact modes (see Fig. 4a,b). The structure enabled us to study the transport of thermally activated electrons between Ti electrodes divided by the tip-induced $\mathrm{TiO}_{x}$. It was a planar MIM-type diode prepared on a Si substrate with a $100 \mathrm{~nm}$ thick $\mathrm{SiO}_{2}$ insulating layer. In our design the Ti layer was $10 \mathrm{~nm}$ thick and $5 \mu \mathrm{m}$ wide in its narrow part (Fig. 4b). The ends of the Ti layer formed source and a drain contacted by Au wires and silver conductive paste. In the narrow part of the layer Ti-oxide lines were formed by AFM in the contact or non-contact modes. To oxidize the layer completely, the lines were overwritten 10 times with low tip speed $(0.05 \mu \mathrm{m} / \mathrm{s})$. The applied voltage was $8 \mathrm{~V}$ in the contact mode and $13 \mathrm{~V}$ in the non-contact mode, and the humidity was $40 \%$. Figure $4 \mathrm{~b}$ shows the image of the central part of the structure with the oxide line prepared in contact mode. 

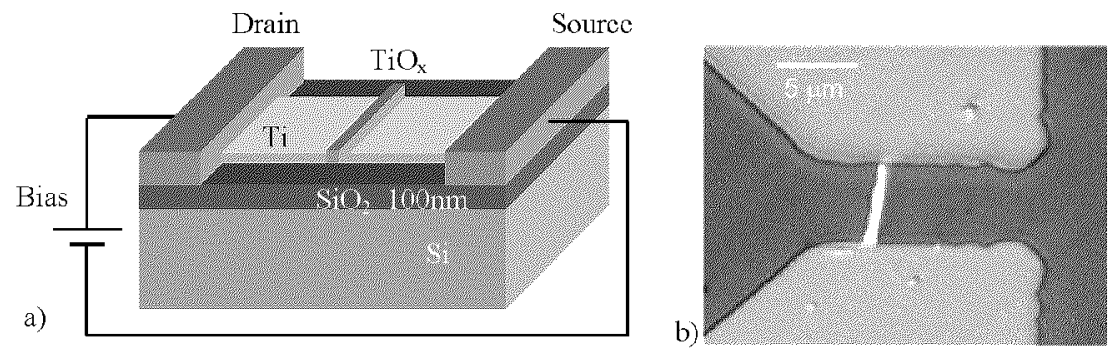

Fig. 4. (a) Planar-type of the metal-insulator-metal diode prepared on Si substrate with $100 \mathrm{~nm}$ thick $\mathrm{SiO}_{2}$ insulating layer and $10 \mathrm{~nm}$ thick Ti layer; (b) central part of the planar MIM diode with the oxide line prepared in the contact mode.

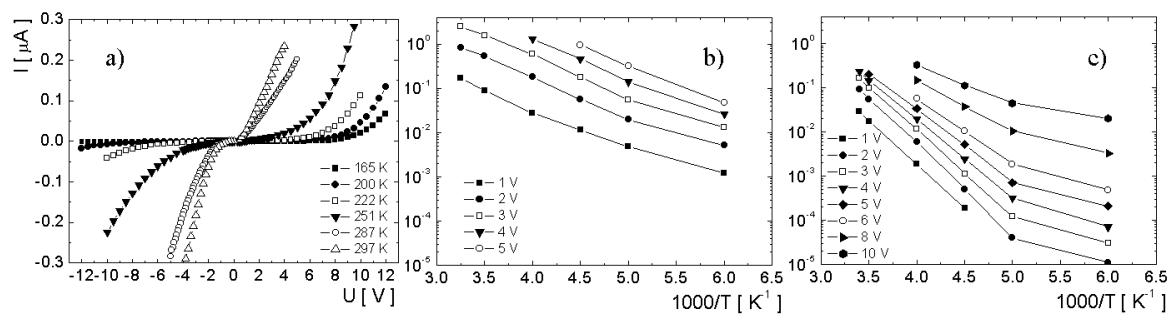

Fig. 5. (a) Typical current-voltage characteristics of the planar MIM diode prepared in the non-contact mode for temperatures from 166 to $297 \mathrm{~K}$; (b) and (c) depict the temperature dependence of the planar current of the MIM diode prepared in contact and non-contact modes, respectively.

Figure 5a shows typical current-voltage characteristics of the planar MIM diode prepared in the non-contact mode for temperatures from 166 to $297 \mathrm{~K}$. The current strongly depends on temperature. The oxidized line represents an energy barrier through which the flowing current is ruled by thermionic-field emission.

Figures $5 \mathrm{~b}$ and $5 \mathrm{c}$ depict the temperature dependence of the planar current of the MIM diode prepared in the contact and in the non-contact modes, respectively. Bias voltage from $1 \mathrm{~V}$ to $10 \mathrm{~V}$ is a parameter. Both graphs show that the transport current, $I$, strongly depends on temperature, $T$, i.e., it is dominated by the transport of thermally activated carriers through a barrier of the height $\Delta E$ (carrier scattering in the barrier is not assumed here, the effective width of the lines is $\approx 100 \mathrm{~nm}$ ). In this case the thermionic emission current is

$$
I \sim \exp (-\Delta E / k T),
$$

where $k$ is the Boltzmann constant.

The slopes of the curves at low voltage and at higher temperatures (308$200 \mathrm{~K}$ ) directly determine the height of the energy barriers. For the lines prepared by contact LAO (Fig. 5b) it is found to be $\approx 200 \mathrm{meV}$, and for the non-contact lines it is $\approx 400 \mathrm{meV}$. 
The difference in the values of $\Delta E$ can be explained by the chemistry of the $\mathrm{TiO}_{x}$ formed using various AFM modes: the oxides obtained by both methods probably differ in their structure and/or composition. The slope decreases for higher voltages. This is probably caused by the local heating of the transport current. The slope decrease in the curve at $166-200 \mathrm{~K}$ is probably caused by the tunneling current through the $\mathrm{TiO}_{x}$ barrier [6].

\section{Conclusions}

We have studied the technological potential of $\mathrm{TiO}_{x}$ mask prepared by local anodic oxidation using atomic force microscope in contact and non-contact modes. It can be concluded that the non-contact mode is not suitable for $\mathrm{TiO}_{x}$ mask preparation for GaAs nanotechnology - the oxide formed is not resistant to several etching solutions commonly used to remove Ti. In contrast, the $\mathrm{TiO}_{x}$ mask prepared in the contact mode is promising for the applications in the AFM nanotechnology and processing.

We have also studied the insulating properties of $\mathrm{TiO}_{x}$ lines prepared in the contact and in the non-contact modes in planar MIM-diode configurations. We show that the effective energy barrier height is $\approx 200 \mathrm{meV}$ for the insulating $\mathrm{TiO}_{x}$ lines prepared in the contact mode, and $\approx 400 \mathrm{meV}$ for the lines prepared in the non-contact mode.

Etching experiments as well as current-temperature characteristics of planar MIM diodes prepared by local anodic oxidation have shown the importance of the AFM mode for the Ti-oxide structure formed.

\section{Acknowledgments}

This work was sponsored by the Slovak APVT-26-020902 project "Submicron vector Hall probe microscope".

\section{References}

[1] S. Lüscher, A. Fuhrer, R. Held, T. Heinzel, K. Ensslin, M. Bichler, W. Wegscheider, Microelectron. J. 33, 319 (2002).

[2] E.S. Snow, D. Park, P.M. Campbell, Appl. Phys. Lett. 69, 269 (1996).

[3] K. Matsumoto, M. Ishii, K. Segawa, Y. Oka, B.J. Vartanian, J.S. Harris, Appl. Phys. Lett. 68, 34 (1996).

[4] S. Lemeshenko, S. Gavrilov, V. Shevyakov, V. Roschin, R. Solomatenko, Nanotechnology 12, 273 (2001).

[5] Y. Mori, N. Watanabe, J. Electrochem. Soc. 125, 1510 (1978).

[6] K. Matsumoto, S. Takahashi, M. Ishi, M. Hoshi, A. Kurokawa, S. Ichimura, A. Ando, Jpn. J. Appl. Phys. 34, 1387 (1995). 